\title{
Haptic feedback improves surgeons' user experience and fracture reduction in facial trauma simulation
}

\author{
Sabine Girod, MD, DDS, PhD; ${ }^{1}$ Sara C. Schvartzman, PhD; ${ }^{2}$ Dyani Gaudilliere, DMD, MPH; ${ }^{1}$ Kenneth Salis- \\ bury, PhD; ${ }^{2}$ Rebeka Silva, DMD ${ }^{3 *}$ \\ Departments of ${ }^{1}$ Surgery, Oral Medicine \& Maxillofacial Surgery Section, and ${ }^{2}$ Computer Science, Stanford University, \\ Stanford, CA; ${ }^{3}$ Dental Service, San Francisco Department of Veterans Affairs Health Care System, San Francisco, CA
}

\begin{abstract}
Computer-assisted surgical (CAS) planning tools are available for craniofacial surgery but are usually based on computer-aided design (CAD) tools that lack the ability to detect the collision of virtual objects (i.e., fractured bone segments). We developed a CAS system featuring a sense of touch (haptic) that enables surgeons to physically interact with individual, patient-specific anatomy and immerse in a threedimensional virtual environment. In this study, we evaluated initial user experience with our novel system compared to an existing CAD system. Ten surgery resident trainees received a brief verbal introduction to both the haptic and CAD systems. Users simulated mandibular fracture reduction in three clinical cases within a $15 \mathrm{~min}$ time limit for each system and completed a questionnaire to assess their subjective experience. We compared standard landmarks and linear and angular measurements between the simulated results and the actual surgical outcome and found that haptic simulation results were not significantly different from actual postoperative outcomes. In contrast, CAD results significantly differed from both the haptic simulation and actual postoperative results. In addition to enabling a more accurate fracture repair, the haptic system provided a better user experience than the CAD system in terms of intuitiveness and self-reported quality of repair.
\end{abstract}

Key words: 3-D user interfaces, bimanual haptics, computerassisted surgical simulation, craniofacial surgery, craniofacial trauma, force feedback, mandible fracture, surgical simulation, virtual environments, visuohaptic.

\section{INTRODUCTION}

Craniofacial injuries are a common occurrence in both civilian and combat trauma. The 2014 annual report from the National Trauma Data Bank database revealed that nearly 24 percent of injuries affect the face [1]. Prior to 1970, motor vehicle accidents were the most frequent cause of facial injuries in the United States. Since then, the number of violence-, job-, and sports-related injuries as well as self-inflicted injuries has risen, contributing to a fairly constant number of facial injuries, despite a decrease in motor vehicle accident-related facial trauma [2-3]. In military operations, an evolving pattern of injuries has been

Abbreviations: $3-\mathrm{D}=$ three dimensional, $6-\mathrm{DOF}=$ six degrees of freedom, $\mathrm{CAD}=$ computer-aided design, $\mathrm{CAS}=$ computerassisted surgical, $\mathrm{CT}=$ computed tomography, $\mathrm{CW}=$ coronoid width, $\mathrm{GW}=$ gonion width, $\mathrm{LCW}=$ lateral condyle width, $\mathrm{MAL}=$ mandibular angle left, $\mathrm{MAR}=$ mandibular angle right, MLL $=$ mandibular length left, MLR $=$ mandibular length right, $\mathrm{OEF}=$ Operation Enduring Freedom, OIF $=$ Operation Iraqi Freedom, $\mathrm{SD}=$ standard deviation.

*Address all correspondence to Dr. Rebeka Silva, San Francisco Department of Veterans Affairs Health Care System, Dental Service (160), 4150 Clement St, San Francisco, CA 94121; 415-750-2046; fax: 415-750-6603.

Email: Rebeka.silva@va.gov

http://dx.doi.org/10.1682/JRRD.2015.03.0043 
noted in Operation Enduring Freedom (OEF) and Operation Iraqi Freedom (OIF). As a result of the effective protection of the torso and skull by Kevlar body armor, the face (below the helmet), the neck, and the limbs account for the majority of injuries [4-5]. One study showed that 21 percent of all battle-injury casualties in OEF and OIF presented with injuries involving the head and neck region [6]. The incidence is reportedly even higher when patients with intracranial neurosurgical injuries and primary ophthalmic injuries are included. In another study, 39 percent of all battle-injury soldiers in OIF II presented with injuries involving the head, face, and neck region [7].

Despite the large and consistent need for craniofacial trauma surgery in both civilian and military settings, it remains one of the most challenging surgical endeavors. With the complex anatomy of the head and neck, the critical functional requirements of the mouth and airway, and the high visibility of the face, even small errors in bone alignment can have major consequences. Thus, successful craniofacial surgeries require precise preoperative diagnostics and planning.

In recent years, virtual computer-assisted surgical (CAS) systems have led to a paradigm shift in surgical planning for patients and training of surgeons [8]. These virtual surgical tools can be used to simulate bone cutting, to remove and move bone segments, and to allow limited prediction of soft tissue changes [9]. Using threedimensional (3-D) anatomical information of traumatic craniofacial injuries from diagnostic computed tomography (CT) imaging, an accurate virtual model of an individual patient can be reconstructed and used for CAS simulation in various applications, including treatment planning, procedure rehearsal, education, and biomechanical loading and stress distribution simulation [10]. These CAS programs enable various treatment alternatives to be simulated and transferred into the operating room using CT-based surgical navigation [11].

Despite the progress in virtual surgical planning, the currently used systems are usually based on computeraided design (CAD) tools with a "windows, icons, menus, pointer" interface of drop-down menus, which insufficiently represent the 3-D and tactile experience of surgery and lack the ability to detect the collision of virtual objects (i.e., fractured bone segments). To achieve both these functions, a haptic (force-feedback) interface could be employed to enable physical contact between the computer and the user through an input/output device; thus, surgeons could train on the procedure by getting tactile feedback whenever virtual bone fragments collided.

We developed a 3-D computational platform that enables surgeons to physically interact with individual, patient-specific anatomy and immerse in a 3-D virtual environment. Our system utilizes high-fidelity haptic devices with six degrees-of-freedom (6-DOF) to allow realistic rehearsal and planning of craniofacial reconstructive surgeries similar to the actual surgery. In this study, using a mandibular fracture model, we compared the user experience of our newly developed haptic system with a known CAD system. We hypothesized that (1) surgery resident trainees would find the haptic system easier and more intuitive to use than the CAD software tool, and (2) the results of the haptic system would more closely resemble the actual surgical outcome than the CAD system simulations.

\section{METHODS}

We selected a well-known CAD system, Maya (Autodesk Inc; San Rafael, California), that allows the user to manipulate virtual objects on the computer monitor through the use of a mouse and keyboard. Maya can enable the manipulation of virtual objects (i.e., irregular pieces of fractured bone) and allow the user to position the fragments as desired. We compared Maya to our newly developed haptic system, which allows force-feedback interaction, including collision detection, with Sensable's Phantom Omni haptic devices (now sold as Geomagic Touch, 3D Systems; Rock Hill, South Carolina). Our system allows the user to manipulate individual bone segments or lock and unlock up to three joined segments to move multisegment units. The virtual bone segments can be rotated with ease, allowing visualization and assessment of the fracture reduction from multiple points of view (Figure 1).

\section{Study Design and Sample}

Participants consisted of 10 volunteers who completed testing and surveys with minimal risk. Inclusion criteria were resident trainee status in either an oral/maxillofacial surgery residency program $(n=8)$ or a plastic surgery residency program $(n=2)$ and no prior experience with either our new haptic system or the Maya CAD system. Eight resident surgeons were male and two were female. The 


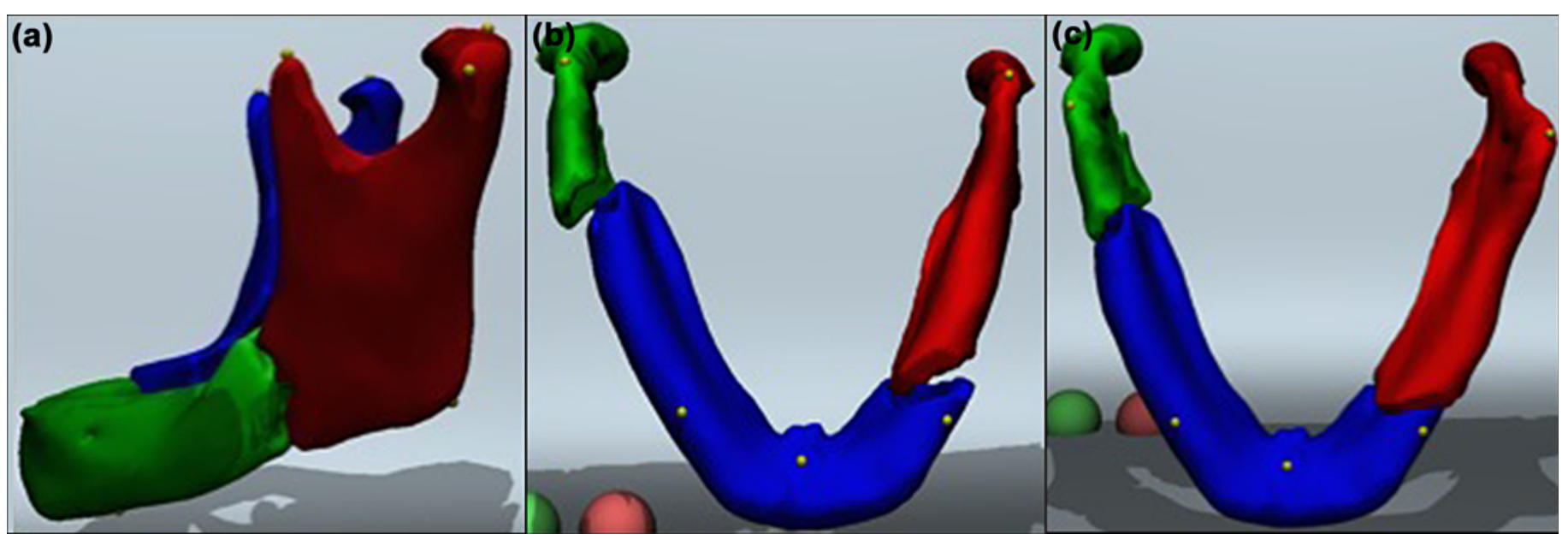

Figure 1.

The haptic computer-assisted surgical interface. (a) Lateral view of the mandible. (b) View of the inferior border of the mandible before virtual fracture reduction. (c) View of the inferior border of the mandible after virtual fracture reduction. The virtual bone segments can be rotated and visualized from multiple points of view. Bone fragments can be "grabbed" and locked with the red and green balls, seen at lower left of (b) and (c), for fracture reduction. The surgeon can feel the collision of the fragments similar to real surgery.

average age was $31.5 \mathrm{yr}$. The time of postgraduate surgical training ranged from 1 to $6 \mathrm{yr}$, with an average of $4.5 \mathrm{yr}$.

The mandible fracture cases presented to each study participant were derived from actual preoperative CT scans from three different patients treated at the San Francisco Department of Veterans Affairs Health Care System by one of the authors (RS). Each of the CT scans was deidentified, and the radiographic data was segmented so that each fractured segment could be manipulated separately. The CT-derived cranial base image was featured for each case to allow the surgery resident to have a fixed point of reference. Case 1 was a denate patient with a right mandibular body fracture. Case 2 was an edentulous patient with a right parasymphyseal fracture and a left angle fracture (Figure 2). Case 3 was an edentulous patient with a bilateral mandibular body fracture with a small defect of missing bone on the left side. Each actual patient underwent postoperative imaging with a CT scan following fracture repair.

Each surgeon received 5 min of verbal instruction on both the Maya software and our haptic system. Following this, the surgeons were presented with the three mandibular fracture cases and asked to manipulate the virtual bone fragments to achieve reduction of the fracture(s). Each study subject was allowed to work for $15 \mathrm{~min}$ for each of the three cases for both the CAD and the haptic system. The order of the cases and the order of whether the CAD or the haptic system was used first were randomized. The outcome measures were (1) accuracy of virtual fracture repair compared with actual postoperative results and (2) quality of the user experience.

\section{Data Collection Methods}

\section{Standard Landmarks and Measurements}

We compared the virtual repairs with the actual postoperative result using radiographic landmarks [12]. Standardized mandibular measurements were recorded using Prism 6.0 (GraphPad; San Diego, California) after all 10 users completed their surgical simulations of the three fracture cases using both systems. Reconstructive hardware, consisting of bone plates and screws, were digitally removed in the actual postoperative CT scans.

The following standard anatomic landmarks and linear and angular measurements were utilized to compare virtual mandibular fracture repairs with the actual postoperative outcomes for each of the three cases as previously described [13]:

1. Anatomic mandibular landmarks:

- Gonion (angle of the mandible).

- Condyle lateral (lateral aspect of the mandibular condyle).

- Coronoid process (tip of the mandibular coronoid process). 


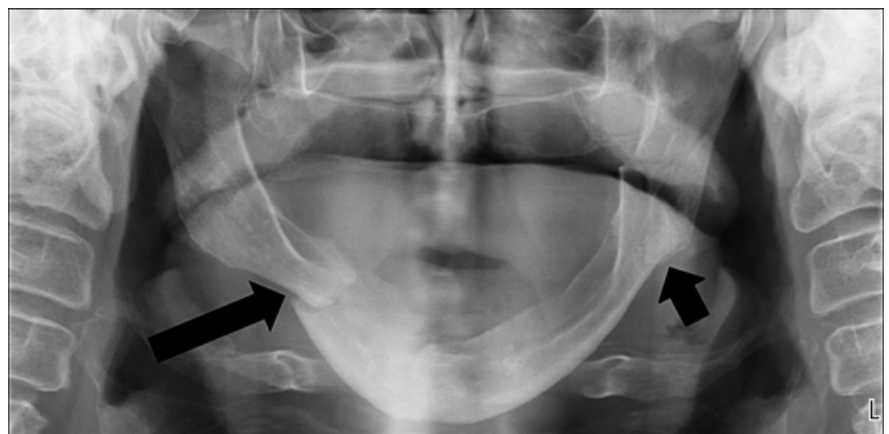

Figure 2.

Panoramic radiographic image of Case 2 showing edentulous mandible with right (long arrow) and left fracture (short arrow).

- Mental foramen (exit point of the mental nerve at the lateral mandibular body).

- Gnathion (most outward point on the profile curvature of the chin).

2. Linear and angular measurements based on these landmarks:
- Mandibular angle left/right (MAL/MAR) (angle between gnathion, gonion, lateral condyle).

- Mandibular length left/right (MLL/MLR) (summed distance between gnathion, mental foramen, gonion).

- Coronoid width (CW) (distance between coronoid process tips).

- Gonion width (GW) (distance between gonions).

- Lateral condyle width (LCW) (distance between lateral condyles).

\section{User Study Questionnaire}

Following the completion of the study (three fracture cases completed using both systems), each study subject completed an online questionnaire designed to assess his or her previous experience using haptic devices, CAD software, and video games. We also assessed the users' experience with the haptic system and the CAD system. Answers were graded on a 5-point Likert scale (Table).

Table.

User questionnaire. The order of the cases and the order of whether the computer-aided design (CAD) or the haptic system was used first were randomized for all study subjects $(N=10)$.

\begin{tabular}{ll}
\hline \multicolumn{1}{c}{ Questions } & Possible Responses \\
\hline General Questions & \\
Age & Male or Female \\
Gender & Year 1-Year 6 \\
What year of post-graduate residency training are you in? & Oral Maxillofacial or Plastics \\
What is your surgical specialty? & 1 (never)-5 (frequently) \\
How often do you play video games? & 1 (no)-5 (frequently) \\
Have you ever used any CAD programs before? & 1 (no)-5 (frequently) \\
Have you ever used any Haptic programs before? & \\
CAD Questions & 1 (poor)-5 (good) \\
How well do you think your fracture repair was in the CAD program? & 1 (one)-5 (all) \\
How many views did you use in the CAD program? & 1 (never)-5 (frequently) \\
Did you feel frustrated when using the CAD program? & 1 (no)-5 (yes) \\
Did you feel that the CAD program was intuitive? & 1 (no)-5 (yes) \\
Did you feel comfortable using the CAD program? & \\
Haptics Questions & 1 (poor)-5 (good) \\
How well do you think your fracture repair was in the Haptic program? & 1 (one)-5 (all) \\
How many views did you use in the Haptic program? & 1 (never)-5 (frequently) \\
Did you feel frustrated when using the Haptic program? & 1 (no)-5 (yes) \\
Did you feel that the Haptic program was intuitive? & 1 (no)-5 (yes) \\
Did you feel comfortable using the Haptic program? & 1 (no)-5 (yes) \\
Did you feel that the force feedback of the Haptic program was helpful? &
\end{tabular}




\section{Statistical Analysis}

Mean and standard deviation (SD) were computed for each of the mandibular measurements for each of the three patients; measures with zero SD (corresponding to segments of the mandible that were not fractured) were excluded. Actual postoperative measurements were used as the control values, and these were compared to the means of the same values for the CAD- and hapticsimulated results using repeated measures one-way analysis of variance. The difference for each measurement was recorded in millimeters or degrees as appropriate. Paired $t$-tests were then used to compare CAD- and haptic-simulated measures to actual postoperative measures and CAD-simulated to haptic-simulated measures. Significance was determined as $p<0.05$.

\section{RESULTS}

\section{Comparison of Virtual Repairs with Actual Postoper- ative Results Using Standard Landmarks}

To analyze the fractured segments of the mandible across the three patients, we included a total of 19 measurements encompassing the following landmarks (described in detail previously): MAR, MLR, CW, GW, and LCW for the first patient; MAL, MAR, MLL, MLR, CW, GW, and LCW for the second patient; and MAL, MAR, MLL, MLR, CW, GW, and LCW for the third patient. For each of these 19 measurements, we compared the mean postsimulated repair values for the 10 participants to their equivalent postsurgical values using paired $t$-tests. Interestingly, we found that the mean of these measurements did not differ significantly between the virtual haptic-simulated repair (mean = 104.50, $\mathrm{SD}=12.29$ ) and the surgical repair (mean $=$ 104.92, $\mathrm{SD}=13.08 ; t(18)=0.83, p=0.42$ ), whereas there was a significant difference between the virtual CADsimulated repair (mean $=102.78, \mathrm{SD}=12.76)$ and the surgical repair $(t(18)=2.64, p=0.02)$. The mean of all measurements was also significantly different between the haptic- and CAD-simulated repairs $(t(18)=3.09, p=$ 0.006) (Figure 3).

We next compared the average absolute value of the differences between the 19 postoperative measurements and their equivalent haptic and CAD measurements using paired $t$-tests. We found that the mean difference of the CAD measurements from the surgical values (mean = $3.16, \mathrm{SD}=2.5)$ was significantly greater than the mean difference of the haptic simulation measurements from the surgical values (mean $=1.79, \mathrm{SD}=3.16)$, in millime-

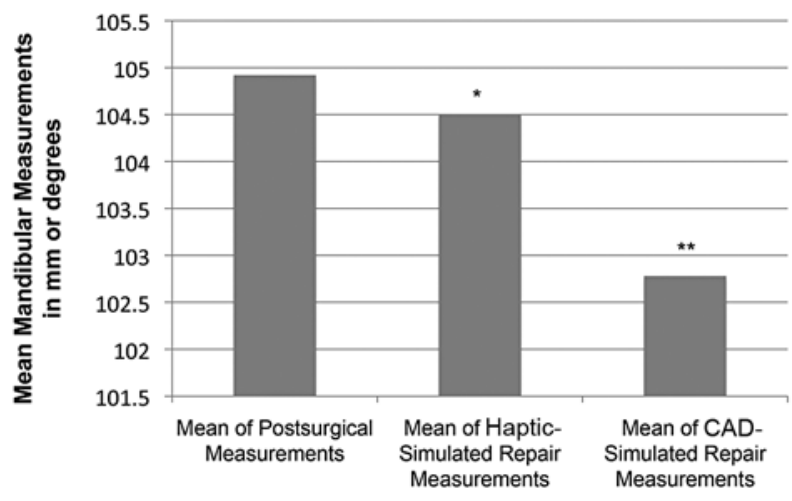

Figure 3.

Mean of 19 mandibular measurements across three patients following simulated repair by 10 participants versus actual surgical repair. CAD = computer-aided design. ${ }^{*}$ There is no significant difference between the haptic-simulated repair and the postsurgical measurements. ${ }^{\star *}$ There is a significant difference between the CAD-simulated repair and the postsurgical measurement.

ters or degrees $(t(18)=-2.41, p=0.03)$. There was also more consistency in the measurements between users for the haptic-simulated repairs than for the CAD-simulated repairs, with a mean SD (determined by calculating the mean SD for each subject's three cases and then calculating the mean SD of all 10 study subjects together for each program) of 2.94 for haptic users and 5.01 for CAD users $(t(18)=-4.87, p \leq 0.001)$.

\section{User Experience Questionnaire}

Following the simulation task, the surgery trainees were asked to complete a questionnaire. We first assessed their previous experience with similar systems. Most users (7 of 10) had never used any haptic or CAD system before. For the other three users, one had prior experience with both haptic and CAD devices, one had prior experience with only CAD programs, and one had prior experience with only haptic systems. The user who had previously used both haptic and CAD systems also frequently played video games, whereas the others rarely (8) or never (1) played video games.

We next evaluated the surgeons' experience with the haptic and CAD systems in this study. The majority of the users (6 of 10) thought that the quality of the haptic fracture reduction was better than the CAD fracture reduction, three thought the fracture reductions were equally good, and one thought the CAD repair was better than the haptic fracture reduction. All participants felt that the haptic force feedback was helpful. 
To compare the user experience between the CAD and haptic systems, we conducted paired $t$-tests for the 5-point Likert scale answers to the questionnaire. Scores for self-reported quality of repair were significantly higher for the haptic system (mean $=3.9, \mathrm{SD}=0.57)$ than the $\mathrm{CAD}$ system $($ mean $=2.9, \mathrm{SD}=1.10 ; t(9)=-2.37, p=0.04)$. Similarly, users rated the intuitiveness of the haptic system (mean $=4.6, \mathrm{SD}=0.52$ ) significantly higher than that of the CAD system $($ mean $=3.1, \mathrm{SD}=0.99 ; t(9)=-4.03, p=$ 0.003 ). Users on average also felt less frustrated and more comfortable using the haptic system (mean $=2.6, \mathrm{SD}=$ 0.97 ; mean $=4.0, \mathrm{SD}=0.94)$ than the CAD system $($ mean $=3.4, \mathrm{SD}=1.35$; mean $=3.2, \mathrm{SD}=1.32)$, though these differences were not significant $(t(9)=-1.81, p=$ $0.10 ; t(9)=-1.71, p=0.12$ ). There was no significant difference between the number of views used in the CAD $($ mean $=4.3, \mathrm{SD}=1.34)$ versus the haptic system (mean $=$ $4.0, \mathrm{SD}=1.33 ; t(9)=0.54, p=0.6$ ) (Figure 4).

We also evaluated whether there were any effects of the order in which the systems were used. There was a higher self-reported intuitiveness of the CAD system for participants who used the haptic system first (mean $=3.8$, $\mathrm{SD}=0.84)$ than those who started with the CAD system $($ mean $=2.4, \mathrm{SD}=0.55 ; t(8)=3.13, p=0.01)$. None of the other self-reported measures were significantly affected by the order of the programs used.

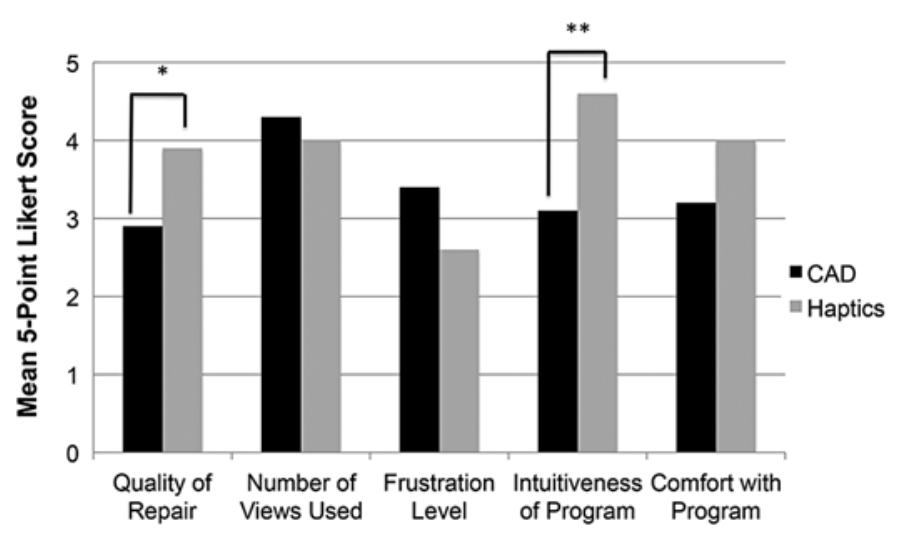

Figure 4.

Self-reported user experience with the haptic system compared with the computer-aided design (CAD) system. A higher score is better for the elements "quality of repair," "intuitiveness of program," and "comfort with program." A lower score is better for "number of views used" and "frustration level." "Significant at $p<0.05$. ${ }^{*}$ Significant at $p<0.01$.

\section{DISCUSSION}

There is a growing demand for patient-specific approaches to complex surgical interventions. Reconstructive craniofacial surgical planning would benefit from the continued development of virtual modeling and simulation environments for surgical planning and education because of the complexity of the anatomy and the visibility and functionality of the face. The benefits of being able to virtually plan craniofacial trauma surgery in the immediate preoperative period are multifold. The surgeon gains preoperative familiarity with the patient's unique bony morphology and the configuration of the fracture segments. Possible surgical solutions can be actively explored, and the surgeon can plan ahead for the type and configuration of the fracture hardware that may be needed in surgery, such as bone plates and screws. Enhanced insight into how the fractured segments fit together would logically lead to improved surgeon confidence and perhaps shorter operating time and/or improved results.

In this study, we tested our newly developed visuohaptic system for direct manipulation of 3-D bone fragment constructs (Figure 1). Our user study revealed that the user friendliness and accuracy of simulated mandibular fracture repairs were superior to a CAD system. The results of the study confirm the notion that interfaces with haptic feedback allow surgeons to perform a mandibular fracture reduction more accurately than with a conventional CAD system in a short time frame (15 min). While both the haptic-based and CAD-based tools allowed surgery resident trainees to plan mandibular trauma repair in the three cases, the virtual surgical repair utilizing the haptic system was more consistent with the actual surgical outcome in all cases tested. The results achieved with the CAD system significantly differed from the actual postoperative results.

In this study, two of the three mandible fracture cases chosen for testing were in edentulous patients. Although measurements between cephalometric landmarks are useful and may be considered a proxy for fracture repair accuracy in edentulous patients, most mandible fractures do occur in patients who have some dentition. Aligning the occlusion between upper and lower teeth is a very important step in mandibular fracture repair; therefore, in such repair, it is a goal to haptically manipulate dentate mandibular fracture segments into precise occlusion with the maxilla. In the early stages of our software development, we 
concentrated on the user interface design and methods of haptically selecting, manipulating, and locking fractured bone segments with collision detection and force feedback. For this reason, this study featured two edentulous mandibular fracture cases.

Other studies have demonstrated that addition of haptic force feedback can enhance the realism of the surgical simulation experience [14]. Moreover, combined hapticvisual training modes have an advantage over either haptic or visual modes alone for tasks that have a force component, such as surgical simulation [15]. However, limitations to surgical training with a haptic feedback simulator were reported in one study; even though haptic feedback enabled a significant improvement in a laparoscopic suturing and knot-tying task with a higher learning rate, the surgeons reached a plateau after $5 \mathrm{~h}$ of training with the haptic device [16]. Recent work by Olsson et al. reported an immersive virtual environment setup with a colocated visuohaptic display and head-tracking capabilities [17]. Their system provides unimanual haptic feedback, secondary hand control for camera movement, and a "snap-to-fit" interaction technique that can assist the surgeon to find an optimal fit of bone fractures. One of the differences between Olsson et al.'s system [17] and the system described here is that ours supports bimanual interaction; empowering both hands improves spatial understanding of the objects and further enhances the similarity to the actual surgical experience. In addition, some commercial haptic devices, including versions of Sensable's Phantom (now sold as Geomagic Touch) and Force Dimension's Delta (Force Dimension; Nyon, Switzerland), are capable of providing 6-DOF forces and torques; for example, Salisbury et al. developed a 6-DOF haptic device designed for surgery-specific applications [18].

The trade-off between haptic performance and model realism can result in an imperfect virtual environment, and thus an imperfect fracture repair plan. However, a purely mathematical approach to automatically find the proper fracture or occlusal alignment would also be prone to ambiguous results. An automatic alignment approach does not take advantage of the surgeon's more global perspective and judgment that is needed to find the best result among competing criteria. Therefore, we feel that integration of the interactive haptic virtual environment with some automatic methods will likely yield the best result in terms of clinical performance. One method to achieve this in future software development is to provide the surgeon with the numerical value of the cost function (metrics) as an additional source of information. Another method is to use the cost function to automatically "snap" a bone fragment or occlusion into place. It is expected that the automated function would only take place when the clinician has already moved the bone fragment(s) and/or occlusion close the desired fixation configuration. To calculate the cost function underlying these methods, we plan to implement the following in the future:

- Marking fracture and dental surfaces: The surgeon can "paint" the fracture surfaces and the surfaces of the teeth using the haptic devices in order to delineate the regions of interest, as proposed by Olsson et al. [17]. With that information, we can generate a numerical value to evaluate whether the fracture surfaces fit together and the occlusion fits together.

- Utilize metrics to quantify quality of fit of the bony reduction and the dental occlusion: There are several ways to evaluate whether the degree of congruence between fracture fragments or between dental occlusal surfaces is close to the desired result. For example, one method is to try to maximize the area of the fractured surface in contact with another fractured surface. Another metric is to minimize the volume between the surfaces. Our aim is to combine these metrics to guide the surgeon in his or her task.

Ultimately, we plan to make our visuohaptic system available online for remote access. Thus, surgeons could access the software to plan and simulate operations independent of their location. Semiautomated features could be integrated into the system to allow the surgeon to rehearse the procedure with expert feedback, especially for less common procedures, prior to the operation and thus shorten procedure times and improve outcomes. A virtual surgery system would also allow off-site surgeons, such as those in combat, to use a realistic and accurate virtual model of their own patient to plan and simulate an operation and evaluate multiple options remotely.

\section{CONCLUSIONS}

Our advanced haptic surgical planning system enabled surgeons to simulate mandibular fracture repair more accurately and with a better user experience than a CAD system. This tool potentially could allow medical students and residents to practice delicate operations and surgeons to plan and rehearse complex procedures and 
maintain their skills for infrequent operations. In the future, other traumatic injuries, such as in civilian or combat orthopedic and plastic surgery, could benefit from the virtual system and computational methods.

\section{ACKNOWLEDGMENTS}

\author{
Author Contributions: \\ Study concept and design: S. Girod, S. C. Schvartzman, K. Salisbury, \\ R. Silva. \\ Acquisition of data: S. C. Schvartzman. \\ Analysis and interpretation of data: S. Girod, D. Gaudilliere, K. Salis- \\ bury, R. Silva. \\ Drafting of manuscript: S. Girod, R. Silva. \\ Critical revision of manuscript for important intellectual content: \\ S. Girod, S. C. Schvartzman, D. Gaudilliere, K. Salisbury, R. Silva. \\ Statistical analysis: D. Gaudilliere. \\ Obtained funding: R. Silva, S. Girod. \\ Study supervision: R. Silva, S. Girod.
}

Financial Disclosures: The authors have declared that no competing interests exist.

Funding/Support: This material was based on work supported in part by the Veterans Health Administration Research and Development Service (award F7124-R), AO Foundation (grant C-10-30G), and the Stanford University Departments of Computer Science and Surgery.

Additional Contributions: S. C. Schvartzman is currently a Research and Development Engineer at MPC, London, United Kingdom.

Institutional Review: The study was approved by the Stanford University Institutional Review Board. All study participants were volunteers and signed an informed consent document.

Participant Follow-Up: The authors do not plan to inform participants of the publication of this study. However, participants have been encouraged to check JRRD for the published article.

\section{REFERENCES}

1. ACS. National Trauma Data Bank 2014 Annual Report [Internet]. Chicago: American College of Surgeons; 2014 [cited 2015 Feb 5]. Available from:

https://www.facs.org/ /media/files/quality\%20programs/ trauma/ntdb/ntdb\%20annual\%20report\%202014.ashx

2. Laski R, Ziccardi VB, Broder HL, Janal M. Facial trauma: A recurrent disease? The potential role of disease prevention. J Oral Maxillofac Surg. 2004;62(6):685-88. [PMID:15170278] http://dx.doi.org/10.1016/j.joms.2003.12.008

3. Gassner R, Tuli T, Hächl O, Rudisch A, Ulmer H. Craniomaxillofacial trauma: A 10 year review of 9,543 cases with 21,067 injuries. J Craniomaxillofac Surg. 2003;31(1):51-61. [PMID:12553928] http://dx.doi.org/10.1016/S1010-5182(02)00168-3
4. Freidlin J, Pak J, Tessler HH, Putterman AM, Goldstein DA. Sympathetic ophthalmia after injury in the Iraq War. Ophthal Plast Reconstr Surg. 2006;22(2):133-34.

[PMID:16550060] http://dx.doi.org/10.1097/01.iop.0000203495.67894.c7

5. Breeze J, Gibbons AJ, Shieff C, Banfield G, Bryant DG, Midwinter MJ. Combat-related craniofacial and cervical injuries: A 5-year review from the British military. J Trauma. 2011;71(1):108-13. [PMID:21336187] http://dx.doi.org/10.1097/TA.0b013e318203304a

6. Xydakis MS, Fravell MD, Nasser KE, Casler JD. Analysis of battlefield head and neck injuries in Iraq and Afghanistan. Otolaryngol Head Neck Surg. 2005;133(4):497-504. [PMID:16213918] http://dx.doi.org/10.1016/j.otohns.2005.07.003

7. Owens BD, Kragh JF Jr, Wenke JC, Macaitis J, Wade CE, Holcomb JB. Combat wounds in Operation Iraqi Freedom and Operation Enduring Freedom. J Trauma. 2008;64(2): 295-99. [PMID:18301189] http://dx.doi.org/10.1097/TA.0b013e318163b875

8. Hsu SS, Gateno J, Bell RB, Hirsch DL, Markiewicz MR, Teichgraeber JF, Zhou X, Xia JJ. Accuracy of a computeraided surgical simulation protocol for orthognathic surgery: A prospective multicenter study. J Oral Maxillofac Surg. 2013;71(1):128-42. [PMID:22695016] http://dx.doi.org/10.1016/j.joms.2012.03.027

9. Meehan M, Teschner M, Girod S. Three-dimensional simulation and prediction of craniofacial surgery. Orthod Craniofac Res. 2003;6(Suppl 1):102-7. [PMID:14606542] http://dx.doi.org/10.1034/j.1600-0544.2003.242.x

10. Goodwin MD, Otake LR, Persing JA, Shin JH. A preliminary report of the virtual craniofacial center: Development of Internet-/Intranet-based care coordination of pediatric craniofacial patients. Ann Plast Surg. 2001;46(5):511-15; discussion 516. [PMID:11352425] http://dx.doi.org/10.1097/00000637-200105000-00010

11. Westendorff C, Gülicher D, Dammann F, Reinert S, Hoffmann J. Computer-assisted surgical treatment of orbitozygomatic fractures. J Craniofac Surg. 2006;17(5):837-42. [PMID: 17003608] http://dx.doi.org/10.1097/01.scs.0000221523.80292.93

12. Whyms BJ, Vorperian HK, Gentry LR, Schimek EM, Bersu ET, Chung MK. The effect of computed tomographic scanner parameters and 3-dimensional volume rendering techniques on the accuracy of linear, angular, and volumetric measurements of the mandible. Oral Surg Oral Med Oral Pathol Oral Radiol. 2013;115(5):682-91. [PMID:23601224] http://dx.doi.org/10.1016/j.0000.2013.02.008

13. Schvartzman SC, Silva R, Salisbury K, Gaudilliere D, Girod S. Computer-aided trauma simulation system with haptic feedback is easy and fast for oral-maxillofacial surgeons to learn and use. J Oral Maxillofac Surg. 2014; 
72(10):1984-93. [PMID:25234531]

http://dx.doi.org/10.1016/j.joms.2014.05.007

14. Pan Z, Cheok AD, Müller W, Zhang X, editors. Transactions on edutainment IV. Berlin (Germany): Springer; 2010. Chapter 19, A framework for virtual hand haptic interaction; p. 229-240.

15. Morris D, Sewell C, Barbagli F, Salisbury K, Blevins NH, Girod S. Visuohaptic simulation of bone surgery for training and evaluation. IEEE Comput Graph Appl. 2006; 26(6):48-57. [PMID:17120913] http://dx.doi.org/10.1109/MCG.2006.140

16. Zhou M, Tse S, Derevianko A, Jones DB, Schwaitzberg SD, Cao CG. Effect of haptic feedback in laparoscopic surgery skill acquisition. Surg Endosc. 2012;26(4):1128-34. [PMID:22044975] http://dx.doi.org/10.1007/s00464-011-2011-8

17. Olsson P, Nysjö F, Hirsch JM, Carlbom IB. A hapticsassisted cranio-maxillofacial surgery planning system for restoring skeletal anatomy in complex trauma cases. Int J Comput Assist Radiol Surg. 2013;8(6):887-94. [PMID:23605116] http://dx.doi.org/10.1007/s11548-013-0827-5

18. Salisbury CM, Salisbury JK, Blevins N, Gillespie RB. A microsurgery-specific haptic device for telerobotic medical treatment. In: Emergency Preparedness and Response and Robotics and Remote Systems Topical Meeting (12th Robotics and Remote Systems for Hazardous Environments/10th Emergency Preparedness and Response); 2008 Mar 11-12; Albuquerque, NM. New York: American Nuclear Society; 2008. p. 404.

Submitted for publication March 19, 2015. Accepted in revised form October 29, 2015.

This article and any supplementary material should be cited as follows:

Girod S, Schvartzman SC, Gaudilliere D, Salisbury K, Silva R. Haptic feedback improves surgeons' user experience and fracture reduction in facial trauma simulation. J Rehabil Res Dev. 2016;53(5):561-70. http://dx.doi.org/10.1682/JRRD.2015.03.0043

ORCID: Rebeka Silva, DMD: 0000-000201789-8086

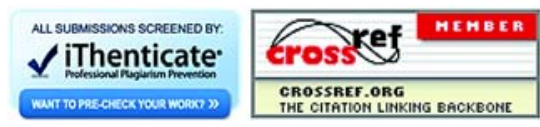


